\title{
CONSTRUÇÃO DO FEMININO E DO MASCULINO: COMPREENSÃO DE UMA EQUIPE DE ENFERMAGEM
}

Laura Ferreira Cortes ${ }^{1}$, Letícia Becker Vieira², Maria Celeste Landerdahl³ , Stela Maris de Mello Padoin ${ }^{4}$

\begin{abstract}
RESUMO: Pesquisa qualitativa objetivou analisar a construção do feminino e do masculino no processo de cuidar de 13 mulheres integrantes de uma equipe de enfermagem de hospital de ensino. A entrevista semiestruturada e vinheta foram aplicadas às profissionais, nos meses de setembro e outubro de 2009. Da análise de conteúdo temática compreendeu-se que as profissionais percebem as diferenças biológicas entre os sexos, mas, sobretudo, a construção social das relações entre homens e mulheres alicerçada na cultura, nos valores, atributos e convenções sociais. Expressam que as instituições sociais, como a família e a Igreja, são responsáveis pela produção e reprodução de masculinidades e feminilidades. Ressalta-se que as participantes, por vezes, não relacionam tal compreensão com o conceito de gênero, reforçando a emergência da inserção de tal conteúdo nos processos de cuidar e na formação.
\end{abstract}

PALAVRAS-CHAVE: Enfermagem; Saúde da mulher; Gênero; Prática profissional.

\section{CONSTRUCTION OF THE FEMININE AND MASCULINE: UNDERSTANDING OF A NURSING TEAM}

ABSTRACT: This qualitative research aimed to analyze the construction of the feminine and the masculine in the care process of 13 female members of a nursing team in a teaching hospital. The semi-structured interview and vignette were applied to the health professionals in September and October 2009. From thematic content analysis, it was understood that the health professionals perceive the biological differences between the sexes but, above all, the social construction of the relations between women and men based in culture, in values, attributes and social conventions. They express that social institutions such as the family and the Church are responsible for the production and reproduction of masculinities and femininities. It stands out that the participants, at times, did not relate this comprehension with the concept of gender, reinforcing the emergence of insertion of such content in the processes of care and training.

KEYWORDS: Nursing; Women's health; Gender; Professional practice.

\section{CONSTRUCCIÓN DEL FEMENINO Y DEL MASCULINO: COMPRENSIÓN DE UN EQUIPO DE ENFERMERÍA}

RESUMEN: Investigación cualitativa que tuvo el objetivo de analizar la construcción del femenino y del masculino en el proceso de cuidar de 13 mujeres integrantes de un equipo de enfermería de hospital de enseñanza. La entrevista semiestructurada y viñeta fueron aplicadas a las profesionales, en los meses de septiembre y octubre de 2009. Del análisis de contenido temático se comprendió que las profesionales percieben las diferencias biológicas entre los sexos, pero, sobre todo, la construcción social de las relaciones entre hombres y mujeres embasada en la cultura, en los valores, atributos y convenciones sociales. Expresan que las instituciones sociales, como la familia y la iglesia, son responsables por la producción y reproducción de cualidades masculinas y femeninas. Se destaca que las participantes, por veces, no relacionan tal comprensión con el concepto de género, reforzando la emergencia de la inserción de ese contenido en los procesos de cuidar y en la formación.

PALABRAS CLAVE: Enfermería; Salud de la mujer; Género; Práctica profesional.

${ }^{1}$ Enfermeira. Residente no Programa de Residência Multiprofissional Integrada em Gestão e Atenção Hospitalar no Sistema Público de Saúde. Membro do Núcleo de Estudos sobre Mulheres, Gênero e Políticas Públicas da Universidade Federal de Santa Maria - NEMGeP UFSM. ${ }^{2}$ Enfermeira. Mestre em Enfermagem. Vice-líder do NEMGeP.

${ }^{3}$ Enfermeira. Mestre em Enfermagem. Doutoranda no Programa DINTER Novas Fronteiras - UFSM/ UNIFESP/ UFRJ. Professora da UFSM. Líder do NEMGeP.

${ }^{4}$ Enfermeira. Doutora em Enfermagem. Professora do Departamento de Enfermagem da UFSM. Líder do Grupo de Pesquisa Cuidado à Saúde das Pessoas, Famílias e Sociedade - UFSM.

Autor correspondente:

Laura Ferreira Cortes

Universidade Federal de Santa Maria

R. Barão do Triunfo, 1623- 97015070 - Santa Maria-RS-Brasil

Recebido: 08/02/11

E-mail: laurafcortez@hotmail.com

Aprovado: 20/05/11 


\section{INTRODUÇÃO}

Concepções sobre gênero têm sido construídas no campo das ciências sociais devido a sua importância como alicerce cultural das relações de poder existentes na sociedade. $\mathrm{O}$ conceito foi utilizado por estudiosos estrangeiros, na década de 60 do século passado. No Brasil, a década de 1980 representou um marco no início de estudos sobre a questão; o conceito foi difundido de forma marcante pelo país a partir de 1990. Com isso foi sinalizada a necessidade de discussão e compreensão do tema na área social e da saúde e paulatinamente introduzido como objeto de pesquisa ${ }^{(1)}$.

A categoria analítica de gênero é considerada a partir de elementos constitutivos das relações sociais entre homens e mulheres e estabelecidos historicamente. O gênero delimita campos de atuação para cada sexo, dá suporte à elaboração de leis e suas formas de aplicação. É construído e alimentado com base em símbolos, normas e instituições que definem modelos de feminilidade e masculinidade, bem como padrões de comportamento, aceitáveis ou não, para homens e mulheres ${ }^{(2)}$. Apreende-se que ser mulher não é apenas diferente de ser homem, mas também que a construção do feminino e masculino é resultante de relações estabelecidas socialmente e que implicam em desigualdades de direitos, inferioridade e opressão.

$\mathrm{Na}$ área da saúde feminina, conquistas e avanços impulsionam políticas públicas, as quais visam superar a visão biologicista de abordagem à mulher, direcionando para a necessidade de considerar as questões de gênero e outros fatores como condicionantes da vida e saúde. Nessa direção, a Organização Pan-Americana da Saúde, em 2000, afirmou que comportamentos de homens e mulheres baseados em padrões hegemônicos de masculinidade e de feminilidade são produtores de sofrimento, adoecimento e morte. No entanto, para as mulheres, esses problemas são agravados pela discriminação nas relações de trabalho, sobrecarga de atividades domésticas e por outras formas de classificação, como etnia, raça, geração e situação de pobreza ${ }^{(3)}$.

As reivindicações das organizações e movimentos de luta pela emancipação das mulheres cada vez mais estimulam aqueles que formulam as políticas nacionais e internacionais a introduzir a questão das desigualdades de gênero entre os pontos importantes a se considerar.

A partir disto, confirmou-se que a subordinação feminina reduz a qualidade de vida das mulheres.
Segundo a Organização das Nações Unidas, as sociedades que buscam maior igualdade de gênero apresentam maior índice de desenvolvimento humano, pois promovem o desenvolvimento econômico e reduzem a pobreza. As mulheres com maior grau de educação e participação no mercado de trabalho, em geral, estão mais capacitadas a contribuir para a saúde e produtividade de suas famílias e localidades, proporcionando melhores perspectivas direcionadas às novas gerações ${ }^{(4)}$.

Na perspectiva de gênero, a Política Nacional de Atenção Integral à Saúde da Mulher/2004 (PNAISM) visa promover a melhoria das condições de vida e saúde das mulheres brasileiras. Está pautada na garantia de direitos e ampliação do acesso aos meios e serviços de promoção, prevenção, assistência e recuperação da saúde da mulher em todo território brasileiro ${ }^{(3)}$. Essas questões estão, também, contempladas no II Plano Nacional de Políticas para as Mulheres/2008 (II PNPM), o qual foi elaborado pela Secretaria Especial de Políticas para as Mulheres, que recomenda a transversalidade de gênero em todas as políticas públicas no Brasil ${ }^{(5)}$.

Aponta-se, então, a necessidade das questões de gênero serem reconhecidas pelos profissionais nas suas práticas assistenciais, no intuito de serem compreendidas como condicionantes de vida e saúde. Vislumbrase, com isto, que a atenção à saúde das mulheres seja permeada pela concepção de integralidade do cuidado, a fim de superar limites na prática profissional e assumir suas responsabilidades no combate às situações de opressão ${ }^{(6-7)}$.

Tais limites se dão em situações cotidianas dos serviços de saúde nas quais os profissionais atuam voltados aos aspectos biológicos. Entretanto, se faz necessário considerar o contexto social e cultural das usuárias dos serviços de saúde. Dentre outras, apontase a prática profissional com mulheres portadoras de doenças sexualmente transmissíveis, na qual, muitas vezes, o profissional não está habilitado e sensibilizado para atuar com questões íntimas e complexas, tais como o contexto de surgimento da doença e a compreensão da relação mulher-parceiro, permeadas pelas questões de gênero $^{(8)}$. Isto contribui para reforçar um sistema patriarcal, de opressão feminina, fortalecendo os entraves para se desenvolver uma assistência integral e humanizada, conforme preconizado pela PNAISM ${ }^{(3)}$.

Nesse contexto, surgem questionamentos sobre o preparo dos profissionais da área da saúde para considerar as desigualdades de gênero na atenção ao público feminino. Percebe-se que as equipes de saúde 
dos serviços

[...] ora constroem espaços de transformação social, ora mantêm a ordem institucional, reproduzindo desigualdades de classe e de gênero na relação profissionalcliente $^{(9: 347) \text {. }}$

Dessa forma, ao considerar que o contexto de iniquidades sociais é subsidiado pelas relações de poder, bem como sua repercussão na saúde das mulheres, fazse necessário ampliar a compreensão do tema entre os profissionais de saúde, em especial os de enfermagem.

Nessa perspectiva, buscou-se analisar como a equipe de enfermagem compreende as questões de gênero no cotidiano de cuidado às mulheres de um do serviço de saúde. O presente artigo constitui-se em parte dos resultados de uma pesquisa e teve como objetivo analisar a construção do feminino e do masculino no processo de cuidar de mulheres de uma equipe de enfermagem em um hospital de ensino.

\section{METODOLOGIA}

Estudo qualitativo, o qual teve como cenário a Unidade Tocoginecológica de um hospital de ensino do interior do Rio Grande do Sul, no Brasil. O uso da abordagem qualitativa justifica-se por esta permitir a busca do universo de significados, motivos, aspirações, crenças, valores e atitudes, o que corresponde a um espaço mais profundo das relações ${ }^{(10)}$.

O critério de inclusão das participantes no estudo foi trabalhar na referida unidade e diretamente no processo de cuidar de mulheres e familiares. A seleção ocorreu de forma aleatória, conforme aceitação e disponibilidade de tempo no momento do convite para a entrevista.

A produção dos dados foi no período de setembro a outubro de 2009, por meio da utilização dos instrumentos de coleta: vinheta e entrevista. Atentou-se para o ambiente da entrevista, sendo este uma sala adequada, no próprio serviço, espaço no qual a entrevistadora e a participante não fossem interrompidas. Naquele momento, era apresentado o termo de consentimento livre e esclarecido e assinando pelas participantes.

A vinheta, técnica utilizada, consistiu em uma descrição curta e compacta de uma situação ou breve evento, real ou fictício, sobre o qual os respondentes são solicitados a refletir e reagir. É estruturada visando extrair informações sobre percepções, para chamar a atenção, passar uma mensagem, produzir sensações e detectar comportamentos, atitudes, opiniões e conhecimentos dos sujeitos acerca do fenômeno investigado.
A vinheta caracteriza-se por ser um meio de obter informações sobre o comportamento das pessoas em situações difíceis de serem observadas na vida diária ${ }^{(11)}$. Nesse estudo, a vinheta foi elaborada com fragmentos da PNAISM, bem como do PNPM ${ }^{(3,5)}$ e seguida da questão: qual sua compreensão sobre as questões de gênero no processo de cuidar de mulheres a partir dos enunciados apresentados?

A entrevista semiestruturada foi composta por seis questões que versavam sobre o tema. Foi aplicada de forma concomitante à vinheta, possibilitando complementação das técnicas, uma vez que as informações da primeira se integravam às da segunda. Desta forma, na medida em que os enunciados da vinheta eram apresentados às participantes, eram feitos os questionamentos que direcionavam para o alcance do objetivo da pesquisa.

A coleta de dados foi encerrada quando houve a convergência dos achados com o objetivo do estudo ${ }^{(12)}$. As entrevistas tiveram tempo médio de duração de trinta minutos, e foram transcritas para análise temática do conteúdo, que se constituiu de três etapas: ordenação, classificação dos dados e análise final, com a composição de categorias temáticas. Para a interpretação procurou-se articular o material estruturado das entrevistas ao referencial teórico do objeto de estudo ${ }^{(10)}$.

As técnicas empregadas foram avaliadas e validadas por docentes doutores, especialistas em metodologia da pesquisa e na temática saúde da mulher; e testadas com profissionais de outro setor do hospital, mostrando-se adequadas aos objetivos propostos.

Os aspectos éticos foram respeitados durante toda a pesquisa e o projeto obteve aprovação do Comitê de Ética em Pesquisa da UFSM sob n. 0119.0.243.000-09. Para a manutenção do sigilo foi utilizado um código com letra a $\mathrm{E}$ (entrevistado) e numeral ordinário em ordem crescente na identificação dos participantes.

\section{RESULTADOS}

Foram entrevistadas seis enfermeiras, seis técnicas e uma auxiliar de enfermagem, totalizando 13 profissionais da equipe de enfermagem.

Quanto ao estado civil, 9 relataram ser casadas e quatro solteiras, com idade entre 21 e 50 anos e média de idade de 35,5 anos. No que diz respeito ao tempo de formação, três profissionais relataram o período de um mês a cinco anos, três profissionais com seis a 10 anos de formação, duas com 11 a 15 anos, duas com 
16 a 20 anos, uma profissional com 21 a 25 anos de formada e uma na faixa de 26 a 30 anos de formação profissional.

$\mathrm{Na}$ análise foram destacadas as palavras que formaram os núcleos de sentidos, compondo três categorias: construção do feminino e do masculino no processo de cuidar de mulheres; compreensão de gênero por meio das implicações na saúde feminina e limites e possibilidades no cotidiano laboral.

Abordaremos neste espaço a categoria "construção do feminino e do masculino no processo de cuidar de mulheres". Para esta foi identificada a compreensão não só de que homens e mulheres são biologicamente diferentes, mas também de que a cultura, a Igreja e as imposições que a sociedade criou determinam o papel social do homem e da mulher. As respondentes delimitaram uma divisão de responsabilidades para os sexos, de o homem sustentar a família e de a mulher ocupar-se com o cuidado da família e filhos e destinada à reprodução. As falas a seguir mostram essa compreensão:

Pra mim é essa diferenciação de sexo mesmo, no homem e na mulher. (E5)

Eu sei que os homens são diferentes das mulheres, os hormônios. (E3)

Eu acho que é cultural. Que nos impuseram isso. Nós vimos essa mãe fazer, essa avó. Isso é cultural. Eu não li nada sobre isso mas, cientificamente, não tem nada que diga que eu tenho que ficar mais tempo com o meu filho que o pai. Isso não está escrito em nenhum lugar. Isso foi uma coisa que a sociedade criou. (E3)

Nós, como mães e mulheres, ainda estamos duplicando aquele modelo antigo. Tu tratas, às vezes, o teu filho, a tua filha, de maneira diferente, inconscientemente nós fizemos isso. E entra também a história da Igreja, que oprimiu muito as mulheres; a Igreja Católica. (E3)

O papel social do homem, ele era responsável por alimentar a família, por sustentar. E essa divisão existiu. Agora, quantos homens trabalham nas atividades domésticas para auxiliar a mulher, ajudam a cuidar dos filhos, participam da gestação, parto e puerpério?! Uma minoria! (E11)

Ao mesmo tempo em que elas estão frequentemente na Unidade de Saúde, muitas vezes, não é para elas, é para outros, para estar cuidando da família. Independente da classe social, é como se ela tivesse essa vestimenta de todas as mulheres terem que desenvolver essa atividade, não é? (E9)

As entrevistadas expressaram, também, a falta de reconhecimento no trabalho, a discriminação em ambiente considerado masculino, situações que foram permeadas por desvalorização do feminino, submissão, desigualdade e dominação pelos homens:

A mulher ainda tem que trabalhar bem mais, estudar bem mais pra ser vista no mercado de trabalho, principalmente. O homem é mais fácil. A mulher tem que se dedicar o dobro pra ser mais reconhecida. (E1)

Quando eu, mulher, chego numa oficina com um automóvel, o mecânico me olha de outro jeito. E quando eu digo assim: "eu quero que você olhe, o meu automóvel, tem isso e isso", eles tentam passar a conversa. Quando tu chegas com um homem, ele te recebe melhor do que se tu chegas sozinha. (E3)

Tanto que quando uma mulher tem outro pensar, ou procura não se envolver tanto nessas questões familiares [cuidado], aí acaba sendo discriminada. A gente percebe uma inferioridade da atitude da mulher, não é? A questão de gênero. Algumas muito submissas também. (E9)

Eu vejo que tem mulheres submissas, que não trabalham fora ainda porque o homem não deixa. (E10)

Os homens se sentem os mandões. Acho que desde que o mundo começou os homens são meio dominadores. Acho que não mudou isso ainda. (E8)

Identifica-se na fala das profissionais a compreensão de que a construção do feminino e do masculino é afirmada por instituições sociais como na família, onde as próprias mulheres reproduzem na educação dos filhos a delimitação do que é ser homem e ser mulher; a opressão das mulheres pela Igreja também é citada. Os atributos delimitados pela construção cultural são responsáveis pelas imagens de masculinidades e feminilidades existentes neste grupo.

\section{DISCUSSÃO}

A construção de gênero tem sido reforçada pela ação conjunta de instituições sociais como a famí- 
lia, a escola, a igreja, a mídia, dentre outros. Estas impõem regras e condutas que determinam comportamentos, espaços, desejos, atividades, sonhos e atitudes de mulheres e homens. Dessa forma, o conceito de gênero é utilizado no sentido de ser não só uma aprendizagem sociocultural, como também para compreender as formas de organização social, discursos e doutrinas ${ }^{(13)}$.

As profissionais deste estudo percebem as diferenças biológicas entre os sexos, mas, sobretudo, a construção social das relações entre homens e mulheres alicerçadas em normas, valores, atributos e convenções sociais. Na ótica de gênero, mulher e homem, feminilidade e masculinidade são definidos por um componente cultural e histórico. Tal conceito é diferenciado de sexo, à medida que considera a mulher e o homem com suas diferenças sociais, ampliando a visão para além da perspectiva biológica ${ }^{(13)}$.

A existência de imagens de masculinidade e feminilidade bem como uma participação insuficiente do homem nas tarefas domésticas e na maternidade são citadas, reforçando a responsabilidade da mulher como mãe, cuidadora do lar, educadora, sendo esta concepção transferida por meio das gerações. Percebese o entendimento, por parte das profissionais, de que existe uma divisão desigual de tarefas entre homens e mulheres. O que converge com estudo também realizado com profissionais de saúde, as quais consideraram que à mulher cabe o espaço privado, as atividades domésticas, a maternidade e o cuidado com a família ${ }^{(14)}$. Neste sentido,

[..] a formação social da mulher foi sendo construída por uma sociedade que ainda a reconhece como um ser inferior em relação à figura masculina e desprestigiada em termos socioculturais ${ }^{(15: 103)}$.

Sendo assim, atribui-se à mulher o cuidar da casa, dos filhos, do companheiro, dos familiares e também das questões de saúde e adoecimento, restringindo seus afazeres, muitas vezes, ao âmbito doméstico.

Pode-se observar que as falas estão permeadas por uma compreensão sobre as relações desiguais entre os segmentos feminino e masculino, de modo que as ações de um e de outro delimitam o que é ser homem e o que é ser mulher. Tal contexto é fruto da construção social que impõe a superioridade do masculino sobre o feminino, gerando desigualdades de poder entre indivíduos e coletivos ${ }^{(1)}$.

As convenções sociais, na maioria das vezes, são reproduzidas pelas próprias mulheres que, desde as primeiras brincadeiras, são condicionadas para o desempenho de papeis como donas-de-casa, esposas e mães. Desse modo, imprime-se na menina a delimitação de seu lugar, o privado, o lugar interno. Para o menino, estimula-se o lugar externo, o oficial, o público ${ }^{(14-15)}$.

O papel feminino, como parte da natureza feminina associado à representação da abnegação e amorosidade, é determinante para que a responsabilidade do cuidado seja delegada à mulher, como por exemplo, o cuidado aos membros do grupo familiar. Cabe-lhe, então, a obrigação de acompanhar os doentes, pais e filhos, nos tratamentos de saúde, eventos da vida social, reuniões escolares, entre outros ${ }^{(14-16)}$.

A naturalização imposta no público e no privado é apontada nas falas das profissionais, de forma que, se houver algum tipo de alteração no desenvolvimento dos comportamentos, previamente estabelecidos, fica evidenciada a discriminação.

No Brasil, a responsabilidade pelo trabalho doméstico ainda é cultural e socialmente atribuída, quase que exclusivamente, às mulheres, que devem desempenhar essas tarefas apenas porque são mulheres ${ }^{(16: 287)}$.

Vislumbra-se, portanto, um ideal de mulher que está aliado ao imaginário da sociedade.

A desvalorização do feminino é percebida como inferioridade e desigualdade, o que decorre do fato de o feminino ser tido como inferior no meio social, à medida que as mulheres dedicam-se, muitas vezes, apenas às atividades domésticas, ou ocupam posições secundárias e subalternas em relação aos homens. Isso acarreta em discriminação e desigualdades no mercado de trabalho, além de representar, para muitas, fragilidade e despreparo para a vida pública ${ }^{(17)}$.

Reforça-se assim, a compreensão por parte das profissionais de saúde do estudo, de que as relações sociais desiguais entre os sexos são permeadas pela submissão ao masculino e às relações de opressão dos homens sobre as mulheres.

As diferenças e desigualdades de gênero estão embutidas nas práticas cotidianas, e encontram legitimidade nos discursos que naturalizam essas diferenças, ou seja, que tomam as propriedades biológicas de homens e mulhres como referências: o forte, o viril, o dominador versus a frágil, a dócil. Mas vale salientar que há possibilidades de mudanças deste paradigma. Apesar de operar lentamente, têm-se os processos de mudanças sociais que são irregulares e perpassam por disposições individuais ${ }^{(4)}$. 
As profissionais do estudo demonstraram, a partir da sua construção do feminino e masculino, uma compreensão para além das questões biológicas, considerando também questões socioculturais. Sem, no entanto, associá-las ao conceito de gênero como categoria de análise das relações que se dão no mundo social.

Vislumbra-se que movimentos de transformação gradativa, com novas concepções e pensamentos que têm alicerçado uma nova visão de mundo dos indivíduos e coletividades como, por exemplo, o Movimento Feminista, a Reforma Sanitária, a PNAISM e o PNPM, possam, na prática, superar o modelo biologicista na saúde. Sendo assim, há a necessidade de inserção do tema na formação acadêmica e permanente nos serviços de saúde. No entanto, este crescimento não se viabiliza do mesmo modo nos currículos dos cursos de formação e pós-graduação na área da saúde, como a Enfermagem ${ }^{(2,18)}$.

\section{CONSIDERAÇÕES FINAIS}

A contribuição das profissionais participantes do estudo está na existência de um entendimento sobre a construção do feminino e do masculino, permeado pela percepção de desigualdades entre mulheres e homens. Contudo, ressalta-se que as profissionais, por vezes, não relacionam tal compreensão com o conceito de gênero, reforçando assim a emergência da inserção de tal pressuposto nas práticas em saúde.

As questões de gênero e suas repercussões na forma como as mulheres cuidam de si e são cuidadas nos serviços de saúde sinalizam as limitações na compreensão das ações preconizadas pela PNAISM. Assinala-se a urgência de discussões na academia e setor saúde sobre a temática, desencadeando o entendimento de suas repercussões na saúde das mulheres brasileiras. Para tanto, repensar o processo de cuidar em saúde e em Enfermagem faz-se necessário, com o intuito de construir competências alinhadas às políticas públicas, e apoiadas em direitos humanos e de cidadania.

Aponta-se como importante o desenvolvimento de discussões e reflexões dos profissionais de saúde, em especial a Enfermagem, sobre masculinidades e feminilidades na perspectiva de gênero considerando as relações sociais.

Recomenda-se o desenvolvimento de outros estudos que criem nexos entre gênero e as implicações no processo de cuidar, contemplando todos os profissionais que compõem a equipe de saúde, e que assistem não só as mulheres como também os homens.

\section{REFERÊNCIAS}

1. Meyer DEE. Gênero: re-construir modos de pensar, ensinar e fazer em saúde e enfermagem. In: Creutzberg M, Funck L, Kruse MHL, Mancia $\mathrm{JR}$, organizadores. Livro-temas do $56^{\circ}$ Congresso Brasileiro de Enfermagem; Enfermagem hoje: coragem de experimentar muitos modos de ser. Gramado(RS)/ Brasília(DF): ABEn; 2005.

2. Gênero: uma categoria útil para a análise histórica. Trad. de Maria Betânia Ávila e Cristine Dabatt. Recife: SOS Corpo; 1989.

3. Ministério da Saúde (BR). Secretaria de Atenção à Saúde. Departamento de Ações Programáticas Estratégicas. Política nacional de atenção integral à saúde da mulher: princípios e diretrizes. Brasília: Ministério da Saúde; 2004.

4. Carrara S. Curso de especialização em gênero e sexualidade. Rio de Janeiro/Brasília: CEPESC/ Secretaria Especial de Política para as Mulheres; 2010.

5. Presidência da República (BR). Secretaria Especial de Políticas para as Mulheres. II Plano Nacional de Políticas para as Mulheres. Brasília (DF): Secretaria Especial de Políticas para as Mulheres; 2008.

6. Coelho EAC, Silva CTO, Oliveira JF, Almeida MS. Integralidade do cuidado à saúde da mulher: limites da prática profissional. Esc Anna Nery. 2009;13(1):154-60.

7. Carvalho C, Destro JR, Faust SB, Coelho EBSC, Boing AF. Dinâmica da violência entre casais a partir da ótica da mulher agredida no bairro Trindade, Florianópolis/ SC. Cogitare Enferm. 2010;15(4):603-8.

8. Araujo LM, Progianti JM, Vargens OMC. A consulta de enfermagem ginecológica e a redução da violência de gênero. Rev Enferm UERJ. 2004;(12):328-31.

9. Coelho EAC. Gênero, saúde e enfermagem. Rev Bras Enferm. 2005;58(3):345-8.

10. Minayo MCS. O desafio do conhecimento: pesquisa qualitativa em saúde. São Paulo: Hucitec; 2007.

11. Polit DF, Hungler BP. Fundamentos da pesquisa em enfermagem: métodos, avaliação e utilização. $5^{\mathrm{a}}$ ed. Porto Alegre: Artmed; 2004.

12. Fontanella BJB, Ricas J, Turato ER. Amostragem por saturação em pesquisas qualitativas em saúde: contribuições teóricas. Cad Saúde Pública. 2008;24(1):17-27. 
13. Figueiredo NMA, Tyrrell MAR. O gênero (in)visível da terceira idade no saber da enfermagem. Rev Bras Enferm. 2005;58(3):330-4.

14. Fonseca RMGS, Galastro EP. A identidade masculina na visão dos profissionais de saúde de um serviço de saúde reprodutiva. REME: Rev Min Enferm. 2006;10(1):3740 .

15. Padoin SMM, Schaurich D. Do cuidado da mulher: questões de gênero e sua incorporação no contexto do HIV/AIDS. Esc Anna Nery. 2004;8(1):101-8.

16. Porto D. Trabalho doméstico e emprego doméstico: atribuições de gênero marcadas pela desigualdade. Rev Bioet. 2008;16(2):287-303.

17. Cabral FB, Ressel LB, Landerdahl MC. Consulta de enfermagem: estratégia de abordagem à gestante na perspectiva de gênero. Esc Anna Nery. 2005;9(3):45965 .

18. Aquino EML. Gênero e saúde: perfil e tendências da produção científica no Brasil. Rev Saúde Pública. 2006;40(n esp):121-32. 